\title{
Role of Leukotrienes in Leukocyte Adhesion Following Systemic Administration of Oxidatively Modified Human Low Density Lipoprotein in Hamsters
}

\author{
H. A. Lehr," C. Hübner,” B. Finckh," S. Angermüller," D. Nolte," U. Beisiegel," A. Kohlschütter," and K. Messmer" \\ ${ }^{*}$ Department of Experimental Surgery, University of Heidelberg, D-6900 Heidelberg, Germany; ${ }^{\ddagger}$ Department of Pediatrics, University of \\ Hamburg, D-2000 Hamburg, Germany; ${ }^{\S}$ Department of Internal Medicine, University of Hamburg; "Department of Anatomy, University \\ of Heidelberg; and 'Institute for Surgical Research, University of Munich, D-8000 Munich, Germany
}

\begin{abstract}
In vitro studies indicate that oxidatively modified low density lipoprotein (oxLDL) promotes leukocyte adhesion to the vascular endothelium, a constant feature of early atherogenesis. Using intravital fluorescence microscopy in the dorsal skinfold chamber model in awake Syrian golden hamsters, we studied whether (a) oxLDL elicits leukocyte/endothelium interaction in vivo, and whether $(b)$ leukotrienes play a mediator role in this event.

Leukocyte/endothelium interaction was assessed in the time course after intravenous injection of native human LDL (4 $\mathrm{mg} / \mathrm{kg}$ body wt) and of oxLDL $\left(7.5 \mu \mathrm{M} \mathrm{Cu}^{++}, 6 \mathrm{~h}, 37^{\circ} \mathrm{C}\right)$ into control hamsters and into hamsters, pretreated with the selective leukotriene biosynthesis inhibitor MK-886 (20 $\mu \mathrm{mol} / \mathrm{kg}$, i.v.). While no effect was seen after injection of native $L D L$, oxLDL elicited an immediate induction of leukocyte adhesion to the endothelium of arterioles and postcapillary venules. Total and differential leukocyte counts suggest that all leukocyte subsets were likewise affected by oxLDL with no specific preference for monocytes. Stimulation of leukocyte adhesion was entirely prevented in inhibitor-treated animals, suggesting the important mediator role of leukotrienes in oxLDL-induced leukocyte/endothelium interaction. (J. Clin. Invest. 1991. 88:914.) Key words: intravital fluorescence microscopy • microcirculation • atherosclerosis $\bullet$ lipoxygenase
\end{abstract}

\section{Introduction}

The activation of circulating leukocytes and their adhesion to the microvascular endothelium in response to various noxious stimuli characterize the first step in the initiation of an acute inflammatory response (1) and the microvascular manifestation of ischemia/reperfusion injury (2). Through the action of degranulation products, adherent leukocytes induce vascular hyperpermeability and contribute to microvascular injury.

The adhesion of leukocytes to the arterial endothelium in areas of increased macromolecular permeability has also been identified as one of the earliest events in atherogenesis (3). Based on epidemiological studies and in vitro experiments, LDL, in particular after oxidative modification, has been asso-

Address reprint requests to Dr. Hans-Anton Lehr, Institute for Surgical Research, University of Munich, Marchioninistrasse 15, D-8000 Munich 70, Germany.

Received for publication 21 November 1990 and in revised form 13 February 1991

J. Clin. Invest.

(c) The American Society for Clinical Investigation, Inc. 0021-9738/91/07/0009/06 \$2.00

Volume 88, July 1991, 9-14 ciated with enhanced leukocyte recruitment and adhesion to the vascular endothelium (4-6).

It has been shown that oxidatively modified LDL (oxLDL) ${ }^{1}$, but not native LDL, stimulates cultured macrophages to generate products of both the cyclooxygenase and the lipoxygenase pathway of arachidonate metabolism (7). Leukotrienes, metabolites of arachidonate produced in the 5-lipoxygenase pathway, initiate many of the vascular actions of oxLDL. Leukotriene $(\mathrm{LT}) \mathrm{B}_{4}$, one of the most powerful chemotactic substances known, stimulates the adhesion of leukocytes to the endothelial lining and their emigration across the endothelial wall (8). The cysteinyl leukotrienes $\mathrm{LTC}_{4}, \mathrm{LTD}_{4}$, and $\mathrm{LTE}_{4}$ have been implicated in the alteration of vascular permeability inducing macromolecular leakage and interstitial edema (9).

These considerations lead us to propose that oxLDL promotes atherogenesis through leukotriene-mediated leukocyte adhesion to the vascular endothelium. We have recently developed an animal model that allows us to investigate leukocyte/ endothelium interaction in response to systemic administration of oxLDL using intravital fluorescence microscopy in the microcirculation of striated muscle in hamsters (10). Using this model and the highly specific leukotriene biosynthesis inhibitor MK-886 (11), which possesses no unspecific antioxidant effects, we demonstrate in this study that leukotrienes play an essential role in mediating the vascular actions of oxLDL.

\section{Methods}

Lipoproteins. Whole blood was collected from healthy human subjects (20-30 yr of age) into tubes containing $1.5 \mathrm{mg}$ dipotassium EDTA $/ \mathrm{ml}$ blood. LDL fractions were isolated by density gradient centrifugation (12). LDL density cut was $\mathrm{d}=1.045-1.065 \mathrm{~g} / \mathrm{ml}$. LDL stock suspensions containing EDTA were stored $\left(4^{\circ} \mathrm{C}\right.$ under argon in the dark) for a maximum of $7 \mathrm{~d}$. Before oxidative modification of LDL, EDTA was removed by chromatography on Sephadex columns (PD-10, Sephadex G-25M; Pharmacia Fine Chemicals, Uppsala, Sweden). Cholesterol content was determined by Cholesterin Monotest (Boehringer Mannheim GmbH, Mannheim, Germany). LDL suspension was diluted by PBS, without $\mathrm{Ca}^{++}$and $\mathrm{Mg}^{++}$, pH 7.3 (Sigma Chemical Co., Deisenhofen, Germany) to reach a final LDL-cholesterol concentration of 0.85 $\mathrm{mg} / \mathrm{ml}$. Autooxidative modification of LDL was achieved by incubation of the $\mathrm{LDL}$ suspension $(1-1.5 \mathrm{ml})$ in $7.5 \mu \mathrm{M} \mathrm{Cu}^{++}\left(\mathrm{CuSO}_{4}, M_{\mathrm{r}}\right.$ 249.68; E. Merck, Darmstadt, Germany) for $6 \mathrm{~h}$ in a water bath at $37^{\circ} \mathrm{C}$ (13). Immediately after oxidative modification, oxLDL was injected intravenously as a bolus into the hamsters via permanent jugular catheters (4 mg LDL-cholesterol/kg body wt). Control experiments were performed by injecting native $\mathrm{LDL}$ prepared without $\mathrm{Cu}^{++}$during incubation.

Biochemical properties of native $L D L$ and ox $L D L . \alpha$-and $\gamma$-tocopherols were measured with HPLC according to the modified method of

1. Abbreviation used in this paper: oxLDL, oxidatively modified LDL. 
Catignani (14) using a $\mathrm{C}_{18}$ reversed phase column $(4 \times 250 \mathrm{~mm}$; particle size $5 \mu \mathrm{m}$ ) with $98: 2$ methanol/ $\mathrm{H}_{2} \mathrm{O}$ as mobile phase. LDL-tocopherol was detected by fluorescence $(\lambda e x=295 \mathrm{~nm}, \lambda e m=325 \mathrm{~nm})$ and quantified by using $\alpha$ - and $\gamma$-tocopherol as a standard. The $\gamma$-tocopherol peak could theoretically represent $\beta$-tocopherol having the same retention time. Dl- $\alpha$-tocopherol acetate (Sigma Chemical Co., Deisenhofen) was taken as an internal standard and determined in parallel on a second channel by UV measurement at $280 \mathrm{~nm}$. Not detectable values were below $0.02 \mu \mathrm{g} \alpha$-tocopherol and below $0.002 \mu \mathrm{g} \gamma$-tocopherol. Fatty acid determinations were performed by capillary gas chromatography as previously described (15). Lipoperoxides were determined according to El-Saadani et al. (16). Electrophoretic mobility of LDL and oxLDL was assessed on $0.83 \%$ agarose gels. The $R_{\mathrm{f}}$ value is defined as the distance of the LDL spot center from the start point divided by the according distance of the bromphenol blue stained solvent front.

Animal model. For intravital fluorescence microscopy of the microvasculature of striated skin muscle, we used the dorsal skinfold chamber preparation in Syrian golden hamsters $(17,18)$. This model allows in the awake animal the quantification of leukocyte/endothelium interaction, microvessel diameter, and red cell velocity in arterioles and postcapillary venules of the striated muscle contained within the observation window.

Operative technique. Syrian golden hamsters (6-8 wk old, weighing 60-80 g) were anesthetized by intraperitoneal injection of pentobarbital $(60 \mathrm{mg} / \mathrm{kg}$ body $\mathrm{wt})$. The entire back of the animals was shaven and two titanium frames were implanted so as to sandwich the extended double layer of the skin. One layer was completely removed in a circular area of $15 \mathrm{~mm}$ in diameter and the remaining layer, consisting of epidermis, subcutaneous tissue, and the thin striated skin muscle was covered with a cover slip incorporated in one of the frames. Indwelling venous and arterial catheters were inserted in the jugular vein and the carotic artery, respectively, passed subcutaneously to the dorsal side of the neck, and sutured to the titanium frames. The animals tolerated the dorsal skinfold chambers well and showed no signs of discomfort. In particular, no effect on sleeping and feeding habits was noticed.

Intravital fluorescence microscopy. A recovery period of 48-72 h between implantation of the observation chamber and the microscopic investigation was allowed to eliminate the effects of anesthesia and surgical trauma on the microvasculature. Muscle arterioles (30-60 $\mu \mathrm{m}$ in diameter) were chosen for investigation, as well as postcapillary and collecting venules (10-60 $\mu \mathrm{m}$ in diameter), since these vessel segments are considered the major site of interaction between leukocytes and the endothelium in response to noxious stimuli (1). Leukocyte/endothelium interaction, vessel diameter, and red cell velocity were assessed in four to six vessel segments per observation chamber in awake animals. The identical vessel segments were examined before and over the time course after injection of native LDL and oxLDL using a computer-controlled stepping motor-driven platform. For visualization of leukocytes, the in vivo fluorescent marker acridine orange was infused intravenously at a constant rate of $0.5 \mathrm{mg} / \mathrm{kg}$ per min. The microscopic images ( $\times 25$ water immersion objective, total magnification: 560$)$ were recorded on videotape and analyzed off-line using a computer-assisted microcirculation analysis system (19). Sticking leukocytes are given as number of cells per square millimeter of vessel surface as calculated from diameter and length of the vessel segment studied $(200 \mu \mathrm{m})$.

Leukotriene inhibition. The highly selective inhibitor of 5-lipoxygenase activation MK-886 (3-[1-(4-chlorobenzyl)-3-t-butyl-thio-5-isopropylindol-2-yl]-2,2-dimethylpropanoic acid) (11) was administered intravenously as a bolus of $20 \mu \mathrm{mol} / \mathrm{kg} 30 \mathrm{~min}$ before injection of oxLDL. Control animals received equivalent volumes of drug vehicle: $1 \%$ hamster albumin (Sigma Chemical Co., Deisenhofen) and $0.9 \%$ benzyl alcohol in $0.9 \%$ saline, $\mathrm{pH} 7.5,21^{\circ} \mathrm{C}$. Effective plasma levels of MK-886 were ascertained by the measurement of LTB $_{4}$ generation in whole blood after ex vivo stimulation with the calcium ionophore A23187 according to Tateson et al. (20).

Electron microscopy. $15 \mathrm{~min}$ after injection of oxLDL, the animals were anesthetized with pentobarbital and fixed by retrograde perfusion through the abdominal aorta for $5 \mathrm{~min}$. The fixation medium con- tained $2.5 \%$ glutaraldehyde and $0.05 \% \mathrm{CaCl}$ in $0.1 \mathrm{M}$ sodium-cacodylate buffer, $\mathrm{pH} 7.4,21^{\circ} \mathrm{C}$. $100-\mu \mathrm{m}$ microsclicer sections were postfixed for $1 \mathrm{~h}$ in $2 \%$ aqueous osmium tetraoxide, dehydrated in graded ethanol, and embedded in Epon 812. Ultrathin sections were counterstained and examined in a Philips EM301 electron microscope.

Statistical analysis. The Mann-Whitney test or the Wilcoxon test with Bonferroni correction was used for calculating $P$ values, as specified in the legends to tables and figures. $P$ values of $<0.05$ were considered significant. Data are given as mean $\pm \mathrm{SD}$.

\section{Results}

Verification of LDL oxidation. The oxidation of $\mathrm{LDL}$ is a complex process during which numerous chemical and functional properties of LDL are altered (21). The degradation of polyunsaturated fatty acids by lipid peroxidation is preceded by the loss of endogenous antioxidants and the formation of lipid peroxides is temporally associated with the degradation of polyunsaturated fatty acids. To verify whether these changes had occurred in the LDL, oxidatively modified under the conditions in our experiment $\left(7.5 \mu \mathrm{m} \mathrm{Cu}{ }^{++}, 6 \mathrm{~h}, 37^{\circ} \mathrm{C}\right)$ we assessed the content of tocopherols, the degradation of polyunsaturated fatty acids, the relative electrophoretic mobility in agarose gels $\left(R_{\mathrm{f}}\right)$, and the formation of lipoperoxides in native LDL and in oxLDL. $\alpha$-tocopherol and $\gamma$-tocopherol, the major antioxidants in LDL (21), which have been observed to disappear within $6 \mathrm{~h}$ under similar incubation conditions (22), dropped from $3.6 \pm 0.8 \mu \mathrm{g} / \mathrm{ml}$ and $0.4 \pm 0.2 \mu \mathrm{g} / \mathrm{ml}$, respectively, to values below the detection limits. The antioxidant capacity being no longer sufficient to protect the polyunsaturated fatty acids from oxidation, the weight percent of total polyunsaturated fatty acids dropped from $43.2 \pm 3.0$ to $13.4 \pm 7.8(P<0.02$, MannWhitney test) and the ratio of saturated to cis-unsaturated fatty acids increased from $0.4 \pm 0.2$ to $1.0 \pm 0.2$ ( $P<0.02$, MannWhitney test). The electrophoretic mobility of the LDL on agarose gel increased (native LDL: $R_{\mathrm{f}}=0.17 \pm 0.08$ vs. oxLDL $R_{\mathrm{f}}$ $=0.70 \pm 0.16, P<0.02$, Mann-Whitney test), reflecting increased negative surface charge (22). Finally, lipid peroxidation was confirmed by the demonstration of increased lipoperoxide formation (native LDL: $0.06 \pm 0.02 \mu \mathrm{mol} / \mathrm{ml}$ vs. oxLDL: $0.31 \pm 0.04 \mu \mathrm{mol} / \mathrm{ml}, P<0.02$, Mann-Whitney test).

Microcirculatory changes after injection of oxLDL. While under normal conditions, represented by the baseline situation, the majority of leukocytes did not interact with the microvascular endothelium, the injection of oxLDL elicited an immediate onset of leukocyte rolling along the endothelial lining and subsequent firm adhesion to the endothelium of arterioles and postcapillary venules, preferentially at vessel bifurcations (Figs. 1 and 2). No increase in leukocyte/endothelium interaction was observed after injection of native LDL (Fig. 2). Differences in the total number of sticking leukocytes between venules and arterioles (Fig. 2) are most likely due to differences in local shear force conditions with low shear forces prevailing in venules and high shear forces in arterioles.

Pretreatment of the animals for $30 \mathrm{~min}$ with the inhibitor of leukotriene biosynthesis, MK-886, prevented the induction of leukocyte adhesion to the microvascular endothelium (Fig. 2). This effect was not due to local microhemodynamic changes since neither blood pressure and heart rate, nor vessel diameter and red cell velocity were affected by MK-886 (data not shown).

Electronmicroscopic findings. The concept that all leukocyte subpopulations were likewise affected by the injection of 

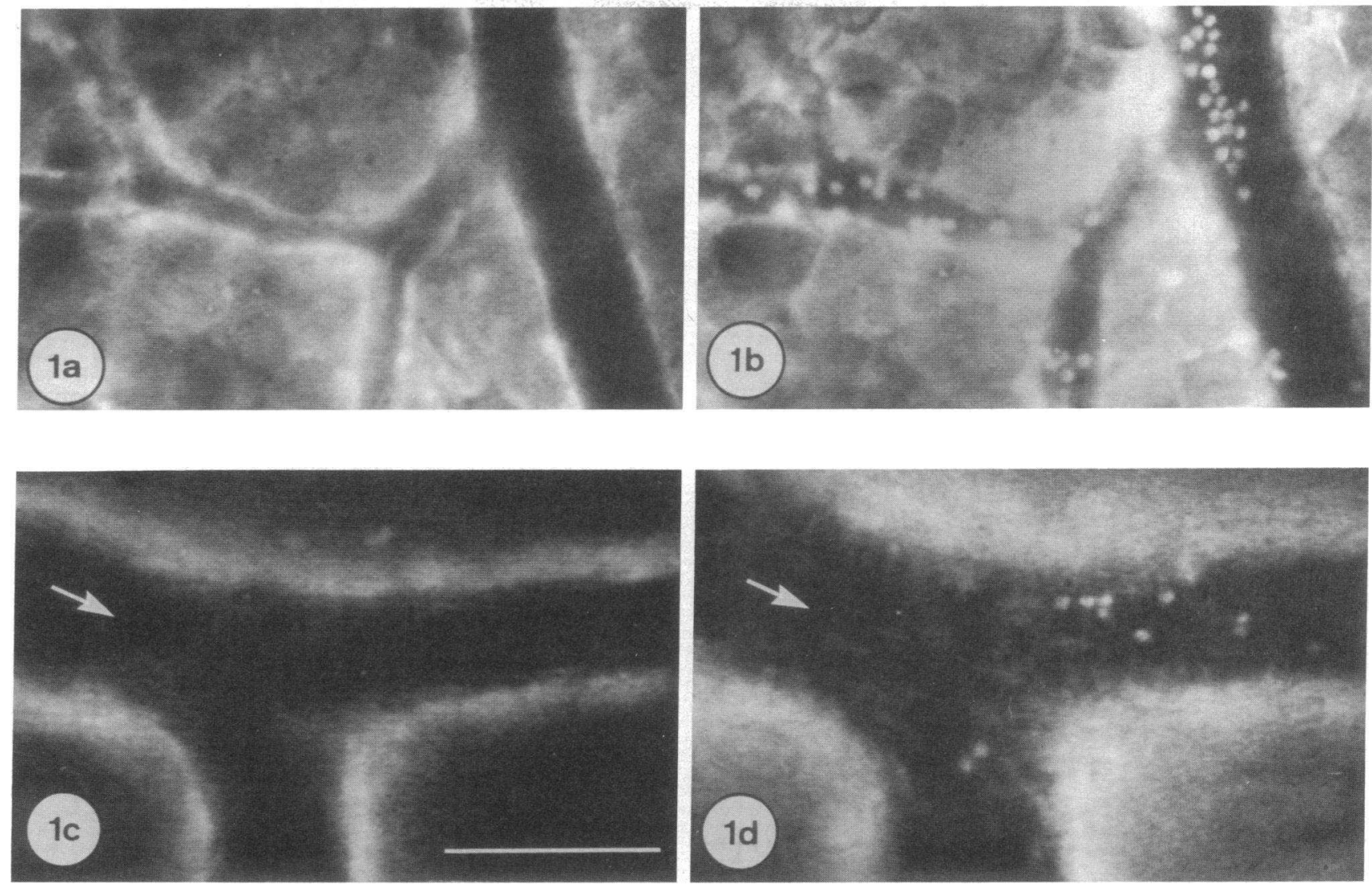

Figure 1. Intravital microscopic demonstration of leukocyte/endothelium interaction after injection of oxLDL. Postcapillary venules $(a, b)$ and arterioles $(c, d)$ of the striated muscle contained within the observation window of the dorsal skinfold chamber in an awake hamster as assessed by intravital fluorescence microscopy. Vessel segments before injection of oxLDL $(4 \mathrm{mg} / \mathrm{kg} ; a, c)$ and $15 \mathrm{~min}$ thereafter $(b, d)$. White dots represent leukocytes stained in vivo with the fluorescent marker acridine orange. Arrows indicate the direction of blood flow in the artery. The space bar designates a length of $100 \mu \mathrm{m}$.
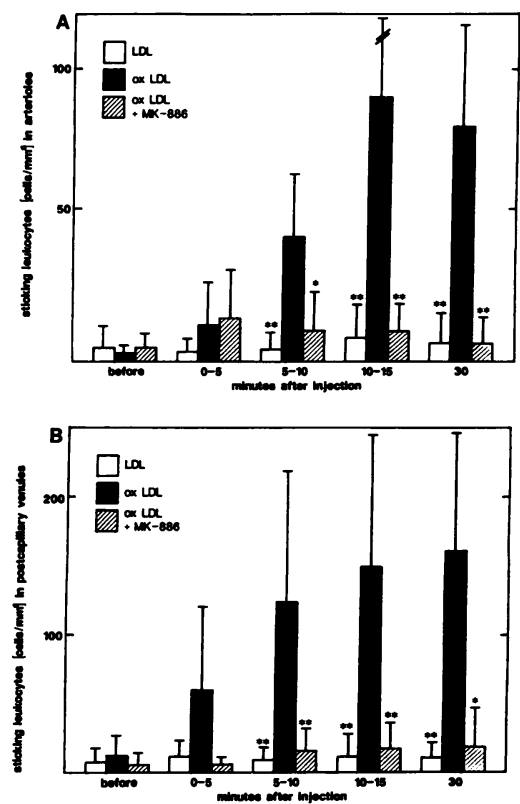
Sticking leukocytes are expressed as number per square millimeter of endothelial surface as the average of all vessel segments per animal. Data given in the figure are the mean $\pm \mathrm{SD}$ of $n=7$ animals. ${ }^{*} P<$ 0.05 and ${ }^{* *} P<0.01$ as compared to corresponding values in vehicletreated animals (Wilcoxon test with Bonferroni correction).
Figure 2. Leukocyte/endothelium interaction in arterioles $(A)$ and postcapillary venules (B) after injection of native LDL and oxLDL. OxLDL (4 mg/kg) was injected intravenously into animals pretreated with MK-886 $(20 \mu \mathrm{mol} /$ $\mathrm{kg}$, i.v., $30 \mathrm{~min}$ before injection of oxLDL; $n$ $=7$, hatched bars) or vehicle ( $n=7$, black bars). Leukocyte/endothelium interaction was quantified in four to six vessel segments per animal before and in the time course after injection of oxLDL. Control alent volumes of native alent volum .

(n)

.
animals received equiv-
oxLDL was substantiated by the electron microscopic observations. Notwithstanding the shortcomings of identifying cells on morphologic grounds alone, cells of all leukocyte subpopulations were found to interact with the endothelium with no evident preference for monocytes (Fig. 3).

Total and differential leukocyte counts. Since acridine orange intercalates with double-stranded DNA and therefore stains all leukocytes without differentiating between the various subpopulations, it is not possible to identify by fluorescence microscopy whether the adherent cells are monocytes, PMNs, or lymphocytes, nor is it possible to discern whether the adherent leukocytes eventually emigrated from the blood stream or reentered the circulation.

To find out whether one or several leukocyte subpopulations were preferentially affected by the adhesion-promoting effect of oxLDL, we performed total and differential leukocyte counts at various times after injection of oxLDL. The total leukocyte count dropped by $34 \% 5 \mathrm{~min}$ after injection and remained at that low level throughout the entire follow-up period (Fig. 4). No changes in total leukocyte count were observed in seven animals following injection of native LDL (data not shown). In the first $10 \mathrm{~min}$ after injection of oxLDL, no changes occurred in differential leukocyte counts, indicating that all leukocyte subpopulations were likewise affected by the adhesion-promoting action of oxLDL (Fig. 4). Starting 20 min and continuing until $120 \mathrm{~min}$ after injection, we observed 

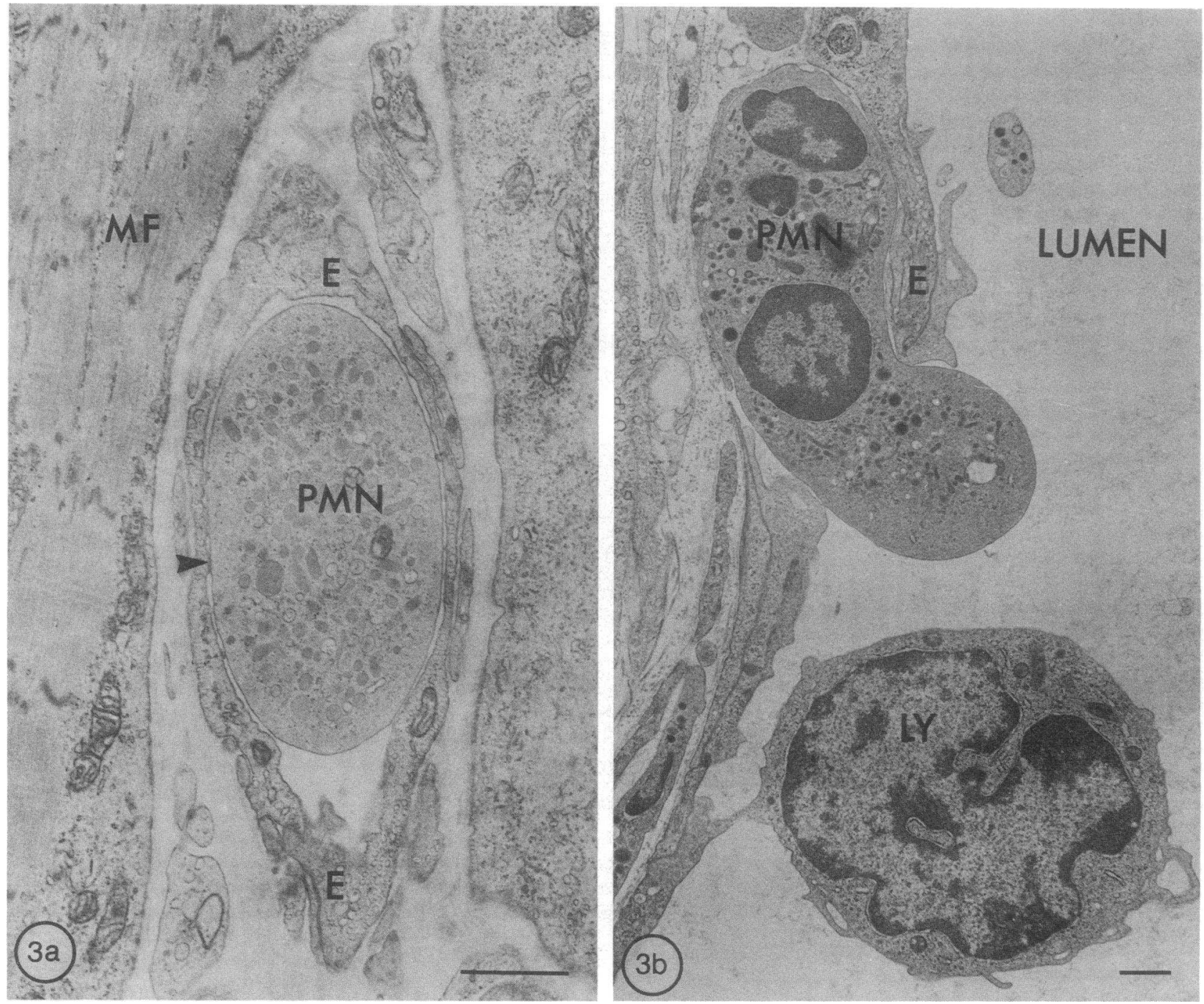

Figure 3. Electron micrographs of leukocyte/endothelium interaction in a capillary (a) and postcapillary venule (b) after injection of oxLDL. (a) Cross-section through a capillary localized in the striated skin muscle $(M F)$ contained within the dorsal skinfold chamber of the hamster, 15 min after injection of oxLDL. The entire lumen of the vessel is plugged by a polymorphonuclear neutrophil (PMN), closely attached to the endothelium $(E)$ of the capillary (arrowhead). $(b)$ Longitudinal section through a postcapillary venule of the subcutaneous connective tissue adjacent to the striated skin muscle. The endothelium is interrupted by the diapedesis of a PMN, extending with a nucleated pseudopod under the attenuated endothelial lamina. Next to the PMN, a lymphocyte $(L Y)$ is exposing discrete spiny processes to take up contact with the endothelium. The space bars designate a length of $1 \mu \mathrm{m}$.

a steady increase in juvenile PMNs presumably in response to the global leukopenia, and a reduction in lymphocytes that might indicate that this leukocyte subpopulation was preferentially retained in the microcirculation (Fig. 4).

The fact that total leukocyte counts remained low over the entire follow-up period suggests that the majority of adherent leukocytes did not reenter the circulation but did emigrate from the blood stream.

\section{Discussion}

The principal result of this study is that intravenous administration of oxLDL elicits leukocyte/endothelium interaction in arterioles and postcapillary venules and that this event depends on 5-lipoxygenase activity.

Leukocyte adhesion to the endothelium at sites of enhanced vascular permeability is a constant finding in long-term cholesterol feeding experiments on various laboratory animals (3). Yet, due to the chronicity of these experiments and the multifactor etiology of atherosclerosis, it was not possible to investigate the effects of single pathogens or mediators on leukocyte/endothelium interaction as the initial event of atherogenesis. This task has been met in the applied model, which allows us to investigate leukocyte/endothelium interaction in both arterioles and postcapillary venules in response to the patho-physiological stimulus oxLDL (10), which has been identified in plasma (23) and atherosclerotic lesions (24). In this study we injected oxLDL that had been oxidized by incubation $\left(37^{\circ} \mathrm{C}\right)$ for $6 \mathrm{~h}$ in $7.5 \mu \mathrm{M} \mathrm{Cu}^{++}$to mimic the modification of LDL as induced by endothelial cells (13). After this period of time, tocopherols had been used and phospholipid peroxidation had resulted in the degradation of polyunsaturated fatty acids and the formation of lipid peroxides.

Intravenous injection of thus modified LDL, but not of 


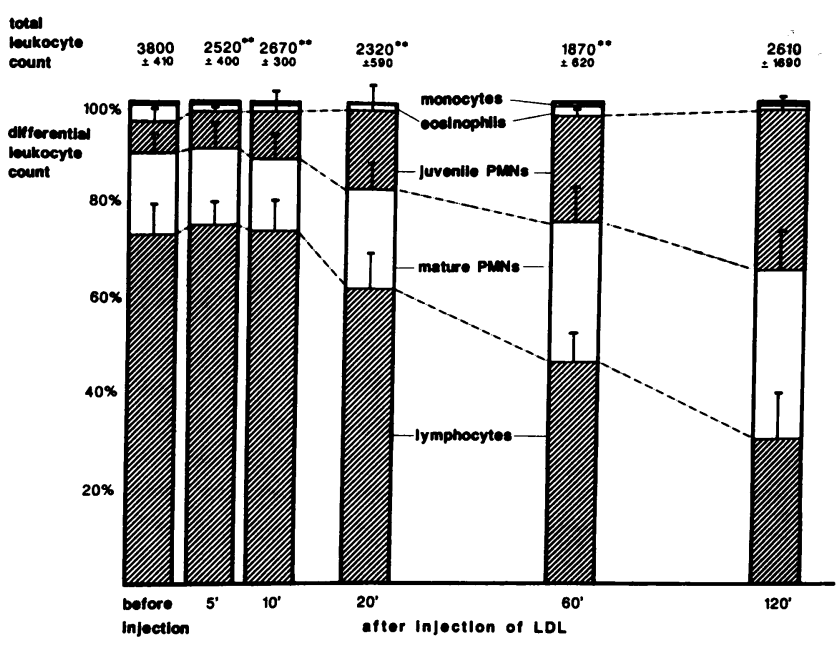

Figure 4. Total and differential leukocyte counts before and in the time course after injection of oxLDL. Data given in the figure are the mean \pm SD of $n=7$ animals. ${ }^{* *} P<0.01$ as compared to baseline values (Wilcoxon test with Bonferroni correction).

identical amounts of native LDL, elicited leukocyte rolling along the endothelial lining within a few minutes after injection, followed by a gradual increase in leukocyte adhesion to the endothelium of both arterioles and postcapillary venules, preferentially at vessel bifurcations (Figs. 1 and 2). This adhesion of leukocytes within the microcirculatory network was associated with a marked drop in total leukocyte count (Fig. 4), suggesting that the phenomenon occurred not only in the investigated muscle tissue, but also in other organs.

It is generally accepted that besides smooth muscle cells, the nonmyogenic cell population in atherosclerotic lesions consists of foam cells of monocytic origin. A chemotactic factor for monocytes produced from endothelial cells after stimulation with oxLDL in vitro has been identified (25). While monocytes differentiate into tissue macrophages and are easily identifiable in histologic sections no morphologic correlate supports the involvement of other nonmyogenic cells in advanced atherosclerotic lesions. On the other hand, cholesterol feeding studies on various laboratory animals showed that not only monocytes, but also lymphocytes and granulocytic cells readily adhered to the vascular endothelium (26). This observation is supported by the results of our study. Although the fluorescence microscopic observations do not allow differentiation of leukocyte subpopulations, differential leukocyte counts in the peripheral blood samples taken before and in the time course after injection of oxLDL suggest that all leukocyte subpopulations were likewise affected by the adhesion promoting action of oxLDL (Fig. 4). This impression is further substantiated by the electron microscopic studies, in which the spectrum of adherent leukocytes correlated well with the differential leukocyte counts in the peripheral blood with no specific preference for monocytes (Fig. 4).

The presence and role of PMNs and lymphocytes in atherogenesis therefore remains a matter of speculation (26). One possible concept is that leukotrienes, metabolites of arachidonic acid generated in the 5-lipoxygenase pathway and released from activated PMNs after their adhesion to the vascular endothelium, contribute to the pathophysiologic sequelae of atherogenesis by propagating the chemotactic accumulation of further leukocytes (8) and by impairing the vascular integrity
(9). This concept could also explain the localization of adherent leukocytes at sites of enhanced permeability (3) and the direct permeabilizing action of oxLDL on the vascular endothelium (27).

The pretreatment of the animals with the selective inhibitor of leukotriene biosynthesis, MK-886 (11), attenuated the provocation of leukocyte/endothelium interaction by oxLDL, suggesting the important role of leukotrienes in this event. MK886 blocks the $\mathrm{Ca}^{++}$-dependent translocation of cytosolic 5-lipoxygenase to its membrane bound activator protein (FLAP) in leukocytes (28) and thus effectively inhibits leukotriene generation in vitro and in vivo (18) without exerting unspecific antioxidant effects (11) (Ford-Hutchinson, Dr. A. W., personal communication). Our in vivo observation extends previous in vitro studies that demonstrate that lysophosphatidylcholine, cleaved from phosphatidylcholine through phospholipase $A_{2}$ activity, promotes the chemotaxis of leukocytes (29), proposing that lysophosphatidylcholine favors the recruitment of leukocytes into the vascular wall during the early stages of atherogenesis in vivo. This concept has been corroborated by studies showing that the antioxidant probucol inhibits LDL oxidation and significantly slows the progression of atherosclerotic lesions in Watanabe heritable hyperlipidemic rabbits (30) (Finckh et al., manuscript in preparation). Yet the subsequent inhibition of phospholipid peroxidation by probucol not only blocks the formation of lysophospholipids, but may also suppress the generation of leukotrienes.

Our idea that oxLDL may promote atherogenesis through the action of leukotrienes is further supported by the demonstration of leukotriene generation in atherosclerotic plaques (31) and by the finding that lipoxygenase inhibitors effectively prevent the uptake of cholesteryl esters into monocytes/macrophages (32).

The mechanism by which oxLDL provoked leukocyte activation and adhesion to the endothelium is incompletely understood. Ishikawa (33) demonstrated that oxLDL stimulates the inositolphosphate turnover in leukocytes to synthesize inositoltriphosphate, a second messenger capable of releasing $\mathrm{Ca}^{++}$ from intracellular stores. Furthermore, oxLDL acts as a $\mathrm{Ca}^{++}$ ionophore, stimulating the influx of extracellular $\mathrm{Ca}^{++}(34)$. Both mechanisms lead to the increase in intracellular $\mathrm{Ca}^{++}$ with subsequent 5-lipoxygenase activation. Another mechanism by which oxLDL may promote leukocyte adhesion is the inhibition of prostacyclin generation (35) in endothelial cells. Since prostacyclin defends against leukocyte adherence to the vascular endothelium (36), it is conceivable that oxLDL-induced reduction of prostacyclin synthesis may promote leukocyte adherence.

Systemic administration of oxLDL creates no chemotactic gradient between the endothelial lining and circulating blood leukocytes. We therefore suggest that oxLDL may not act as a chemoattractant but via the upregulation of adherence receptors, either directly as shown for HLA-DR molecules (6) or indirectly via $\mathrm{LTB}_{4}$-mediated upregulation of $\mathrm{CD} 11 / \mathrm{CD} 18$ adherence receptors (37). This would also explain why not only monocytes, but also PMNs and lymphocytes that also bear this receptor on their surface readily interact with the endothelium following the administration of oxLDL (Figs. 3 and 4).

It has been suggested that atherogenesis in response to vascular injury shares many pathophysiologic mechanisms with the inflammatory response, like leukocyte adhesion and emigration, and increased microvascular permeability (38). Although caution should be exercised in extrapolating the results 
from our acute experiments to the chronic process of atherogenesis, the identification of the involvement of leukotrienes in leukocyte/endothelium interaction as the initial event in atherogenesis stresses the similarities between the two processes and may open the way for novel therapeutic strategies aimed at the prevention of lesion inception and lesion progression.

\section{Acknowledgments}

The authors would like to thank Dr. A. Guhlmann and J. Müller for $\mathrm{LTB}_{4}$ measurements. The excellent technical assistance of G. Rothkegel with intravital fluorescence microscopy and blood work, B. Sehringer-Mansour and I. Wernicke with LDL preparation, and I. Frommer with electron microscopy is gratefully acknowledged. MK886 was kindly provided by Dr. A. W. Ford-Hutchinson, Merck Frosst, Canada.

This study was supported in part by the Deutsche Forschungsgemeinschaft through Sonderforschungsbereich 320 and by the Forschungsschwerpunkt Transplantation Heidelberg.

\section{References}

1. Grant, L. 1973. The sticking and emigration of white blood cells in inflammation. In The Inflammatory Process. L. Grant, B. W. Zweifach, and R. T. McCluskey, editors. Academic Press, New York. 205-249.

2. Mullane, K. M., J. A. Salmon, and R. Kraemer. 1987. Leukocyte-derived metabolites of arachidonic acid in ischemia-induced myocardial injury. Fed. Proc. 46:2422-2433.

3. Ross, R. 1986. The pathogenesis of atherosclerosis-an update. N. Engl. J. Med. 314:488-500.

4. Quinn, M. T., S. Parthasarathy, L. G. Fong, and D. Steinberg. 1987. Oxidatively modified low density lipoproteins: a potential role in recruitment and retention of monocyte/macrophages during atherogenesis. Proc. Natl. Acad. Sci. USA. 84:2995-2998.

5. Steinberg, D., S. Parthasarathy, T. E. Carew, J. C. Khoo, and J. L. Witztum. 1989. Beyond cholesterol. Modifications of low-density lipoprotein that increase its atherogenicity. N. Engl. J. Med. 320:915-924.

6. Frostegard, J., J. Nilsson, A. Haegerstrand, A. Hamsten, H. Wigzell, and M. Giglund. 1990. Oxidized low density lipoprotein induces differentiation and adhesion of human monocytes and the monocytic cell line U937. Proc. Natl. Acad. Sci. USA. 87:904-908.

7. Yokode, M., T. Kita, Y. Kikawa, T. Ogorochi, S. Narumiya, and C. Kawai. 1988. Stimulated arachidonate metabolism during foam cell transformation of mouse peritoneal macrophages with oxidized low density lipoprotein. J. Clin. Invest. 81:720-729.

8. Dahlén, S. E., J. Björk, P. Hedqvist, K. E. Arfors, S. Hammarström, J. A Lindgren, and B. Samuelsson. 1981. Leukotrienes promote plasma leakage and leukocyte adhesion in postcapillary venules: in vivo effects with relevance to the acute inflammatory response. Proc. Natl. Acad. Sci. USA. 78:3887-3891.

9. Hua, X. Y., S. E. Dahlén, J. M. Lundberg, S. Hammarström, and P. Hedqvist. 1985. Leukotrienes $C_{4}, D_{4}$ and $E_{4}$ cause widespread and extensive plasma extravasation in the guinea pig. Naunyn-Schmiedeberg's Arch. Pharmacol. 330:136-141.

10. Lehr, H. A., C. Hübner, D. Nolte, B. Finckh, U. Beisiegel, A. Kohlschütter, and K. Messmer. 1991. Oxidatively modified human low density lipoprotein stimulates leukocyte adherence to the microvascular endothelium in vivo. Res. Exp. Med. 191:85-90.

11. Gillard, J., A. W. Ford-Hutchinson, C. Chan, S. Charleson, D. Denis, A. Foster, R. Fortin, S. Leger, C. S. McFarlane, H. Morton, et al. 1989. L-663,536 (MK-886) (3-1-(4-chlorobenzyl)-3-t-butyl-thio-5-isopropylindol-2-yl) 2,2-dimethylpropanoic acid), a novel, orally active leukotriene biosynthesis inhibitor. Can. J. Physiol. Pharmacol. 67:456-464.

12. Redgrave, T. G., D. C. K. Roberts, and C. E. West. 1975. Separation of plasma lipoproteins by density-gradient ultracentrifugation. Anal. Biochem. 65:42-49.

13. Steinbrecher, U. P., S. Parthasarathy, D. S. Leake, J. L. Witztum, and D. Steinberg. 1984. Modification of low density lipoprotein by endothelial cells involves lipid peroxidation and degradation of low density lipoprotein phospholipids. Proc. Natl. Acad. Sci. USA. 81:3883-3887.

14. Catignani, G. L. 1986. An HPLC method for the simultaneous determination of retinol and a-tocopherol in plasma or serum. Methods Enzymol. 123:215219.

15. Hübner, C., S. G. Lindner, M. Stern, M. Claussen, and A. Kohlschütter. 1988. Membrane fluidity and lipid composition of rat small intestinal brush border membranes during postnatal maturation. Biochim. Biophys. Acta. 939:145-150.

16. El-Saadani, M., H. Esterbauer, M. El-Sayed, A. Y. Nassar, and G. Jürgens. 1989. A spectrophotometric assay for lipid peroxides in serum lipoproteins using a commercially available reagent. J. Lipid Res. 30:627-630.

17. Endrich, B., K. Asaishi, A. Götz, and K. Messmer. 1980. Technical report -a new chamber technique for microvascular studies in unanesthetized hamsters. Res. Exp. Med. 177:125-134.

18. Lehr, H. A., A. Guhlmann, D. Nolte, D. Keppler, and K. Messmer. 1991. Leukotrienes as mediators in ischemia-reperfusion injury in a microcirculation model in the hamster. J. Clin. Invest. 87:2036-2041.

19. Zeintl, H., F. U. Sack, M. Intaglietta, and K. Messmer. 1989. Computer assisted leukocyte adhesion measurement in intravital microscopy. Int. J. Microcirc. Clin. Exp. 8:293-302.

20. Tateson, J. E., R. W. Randall, C. H. Reynolds, W. P., Jackson, P. Bhattacherjee, J. A. Salmon, and L. G. Garland. 1988. Selective inhibition of arachidonate 5-lipoxygenase by novel acetohydroxamic acids: biochemical assessment in vitro and ex vivo. Br. J. Pharmacol. 94:528-539.

21. Esterbauer, H., M. Dieber-Rotheneder, G. Waeg, G. Stiegl, and G. Jürgens. 1990. Biochemical, structural, and functional properties of oxidized low density lipoprotein. Chem. Res. Toxicol. 3:77-92.

22. Jürgens, G., H. F. Hoff, G. M. Chisholm, and H. Esterbauer. 1987. Modification of human serum low density lipoprotein by oxidation-characterization and pathophysiological implications. Chem. Phys. Lipids. 45:315-336.

23. Avogaro, P. G. Bittolo Bon, and G. Cazzolato. 1988. Presence of a modified low density lipoprotein in humans. Arteriosclerosis. 8:79-87.

24. Ylä-Herttuala, S., W. Palinski, M. E. Rosenfeld, S. Parthasarathy, T. E. Carew, S. Butler, J. L. Witztum, and D. Steinberg. 1989. Evidence for the presence of oxidatively modified low density lipoprotein in atherosclerotic lesions of rabbit and man. J. Clin. Invest. 84:1086-1095.

25. Berliner, J. A., M. C. Territo, A. Sevanian, S. Ramin, J. A. Kim, B. Bamshad, M. Esterson, and A. M. Fogelman. 1990. Minimally modified low density lipoprotein stimulates monocyte endothelial interactions. J. Clin. Invest. 85:1260-1266

26. Trillo, A. A. 1982. The cell population of aortic fatty streaks in African Green monkeys with special reference to granulocytic cells. An ultrastructural study. Atherosclerosis. 43:259-275.

27. Morel, D. W., J. R. Hessler, and G. M. Chisholm. 1983. Low density lipoprotein cytotoxicity induced by free radical peroxidation of lipid. J. Lipid Res. 24:1070-1076.

28. Rouzer, C. A., A. W. Ford-Hutchinson, H. E. Morton, and J. W. Gillard. 1990. MK-886, a potent and specific leukotriene biosynthesis inhibitor blocks and reverses the membrane association of 5-lipoxygenase in ionophore-challenged leukocytes. J. Biol. Chem. 265:1436-1442.

29. Quinn, M. T., S. Parthasarathy, and D. Steinberg. 1988. Lysophosphatidylcholine: a chemotactic factor for human monocytes and its potential role in atherogenesis. Proc. Natl. Acad. Sci. USA. 85:2805-2809.

30. Carew, T. E., D. C. Schwenke, and D. Steinberg. 1987. Antiatherogenic effect of probucol unrelated to its hypocholesterolemic effect: evidence that antioxidants in vivo can selectively inhibit low density lipoprotein degradation in macrophage-rich fatty streaks and slow the progression of atherosclerosis in the Watanabe heritable hyperlipidemic rabbit. Proc. Natl. Acad. Sci. USA. 84:77257729.

31. De Caterina, R., A. Mazzone, D. Giannessi, R. Sicari, W. Pelosi, G. Lazzerini, A. Azzara, R. Forder, F. Carey, D. Caruso, et al. 1988. Leukotriene B production in human atherosclerotic plaques. Biomed. Biochim. Acta. 47:S182S185.

32. Van der Schroeff, J. G., L. Havekes, A. M. Weerheim, J. J. Emeis, and B. J. Vermeer. 1985. Suppression of cholesteryl ester accumulation in cultured human monocyte-derived macrophages by lipoxygenase inhibitors. Biochem. Biophys. Res. Commun. 127:366-372.

33. Ishikawa, Y., Y. Asaoka, T. Taniguchi, M. Tsunemitsu, and H. Fukuzaki. 1989. Phosphatidylinositol turnover in human monocyte-derived macrophages by native and acetyl LDL. FEBS (Fed. Eur. Biochem. Soc.) Lett. 246:35-38.

34. Sachinidis, A., R. Locher, T. Mengden, and W. Vetter. 1990. Low density lipoprotein elevates intracellular calcium and $\mathrm{pH}$ in vascular smooth muscle cells and fibroblasts without mediation of LDL receptors. Biochem. Biophys. Res. Commun. 167:353-359.

35. Gryglewski, R. J., and A. Szczeklik. 1978. Inhibition of prostacyclin formation by lipid peroxides in the arterial wall: hypothetical step in development of atherogenesis. Mater. Med. Pol. 10:338-341.

36. Simpson, P. J., J. Mickelson, J. C. Fantone, K. P. Gallagher, and B. R. Luchesi. 1987. lloprost inhibits neutrophil function in vitro and in vivo and limits experimental infarct size in canine heart. Circ. Res. 60:666-673.

37. Tonnesen, M. G., D. C. Anderson, T. A. Springer, A. Knedler, N. Avdi, and P. M. Henson. 1989. Adherence of neutrophils to cultured human microvascular endothelial cells. Stimulation by chemotactic peptides and lipid mediators and dependence upon the Mac-1, LFA-1, p150, 95 glycoprotein family. J. Clin. Invest. 83:637-646.

38. Munro, J. M., and R. S. Cotran. 1988. Biology of disease. The pathogenesis of atherogenesis: atherogenesis and inflammation. Lab. Invest. 58:249-261. 